\title{
The Effect of Magnesium Sulfate on Motor and Sensory Axillary Plexus Blockade
}

\author{
Mohammad Haghighi '; Mehran Soleymanha ${ }^{2}$; Abbas Sedighinejad ${ }^{1}$; Ahmadreza \\ Mirbolook $^{2, *} ;$ Bahram Naderi Nabi $^{1}$; Mehdi Rahmati ${ }^{3}$; Nasim Ashoori Saheli ${ }^{1}$ \\ ${ }_{2}^{1}$ Anesthesiology Research Centre, Guilan University of Medical Sciences, Rasht, Iran \\ ${ }^{2}$ Orthopedic Research Center, Guilan University of Medical Sciences, Rasht, Iran \\ ${ }^{3}$ Anesthesiology Department, Intensive Care Unit Ward, Erfan Private Hospital, Rasht, Iran \\ ${ }^{*}$ Corresponding author: Ahmadreza Mirbolook, Orthopedic Research Center, Guilan University of Medical Sciences, Rasht, Iran. Tel: +98-1313223970, +98-1313228842, E-mail: ahmadre- \\ za.mirbolook@yahoo.com
}

Received: July 11, 2014; Revised: October 21, 2014; Accepted: November 26, 2014

\begin{abstract}
Background: Magnesium is a physiologic cation that blocks neuromuscular transmission and does not allow the nerve to be stimulated. Objectives: This study investigates the effect of adding magnesium sulfate to lidocaine to extend the duration of sensory and motor blocks of the axillary plexus in orthopedic surgeries of the upper extremities.

Patients and Methods: This controlled randomized double-blind study was performed on patients who were candidates for orthopedic surgery of the upper extremities. A total of 60 patients between 18-60 years with ASA Class 1 or 2 participated in the study. One group received lidocaine $(5 \mathrm{mg} / \mathrm{kg}$ ) with magnesium sulfate $20 \%(3 \mathrm{~mL})$ as the case group, while the second group received lidocaine (5 mg/kg) with normal saline $(3 \mathrm{~mL})$ as a placebo to block the axillary plexus using the trans-arterial technique. The duration of the sensory and motor block of the axillary plexus was monitored and evaluated using the pinprick and modified Bromage scale.

Results: A total of 60 patients were included in the study with 30 patients having received lidocaine plus magnesium and the other 30 patients having received lidocaine plus normal saline. The mean sensory block duration in the case group was $248.83 \pm 18.36$ and in control group was 204.67 \pm 22.62 . The mean motor block duration in the case group was $207.0 \pm 16.64$ and in control group was $147.33 \pm 21.52$ (both $\mathrm{P}<0.0001)$. The mean onset of sensory block in case group was $15.5 \pm 3.79$ and the onset block in control group was 10.33 $\pm 4.13(\mathrm{P}<0.0001)$. The mean onset of motor block in case group was $20.66 \pm 4.09$ and the onset block in control group was $19.73 \pm 26.18(\mathrm{P}<0.848)$.

Conclusions: The addition of magnesium sulfate to lidocaine increased the duration of motor and sensory axillary block in the upper extremities during surgeries when compared to the use of lidocaine alone.
\end{abstract}

Keywords:Magnesium Sulfate; Lidocaine; Regional anesthesia

\section{Background}

Regional anesthesia has been accepted as a safe and effective method for various surgical procedures including upper limb surgery. This method can prolong analgesia during surgery and assist with postoperative pain management. An axillary plexus block is commonly used as a regional anesthesia in the hand, arm, and forearm surgeries. Several attempts have been made to prolong the effect of regional anesthetics by sensory and motor blocks of axillary plexus using various factors such as narcotics, verapamil, clonidine, and tramadol (1).

Magnesium sulfate does not let catecholamines be released from the adrenal and peripheral nerve endings. Therefore, catecholamines receptors are blocked. So Magnesium sulfate results are sympathetic blocks (2). Some studies have demonstrated that the magnesium seems to reduce the postoperative pain, moreover injecting intravenously led to a reduction of the consumption of anesthetics during surgery (3). Studies have also shown that intrathecal administration of magnesium can strengthen the analgesic properties of opioids used for pain management following knee arthroscopy and during childbirth labor (4).

\section{Objectives}

Magnesium may use different mechanisms to prolong the effect of regional anesthetics. Our study was done to evaluate the effect of magnesium sulfate with lidocaine when used for sensory and motor axillary-plexus blocks.

\section{Patients and Methods}

This randomized controlled, double blind study was done on 60 patients, who were ASA Class 1 or 2 and between the ages of 18 and 60 . The patients were admitted to the trauma center of Poursina Hospital in Rasht, Iran. In addition, they were candidates for hand and forearm surgery. The duration of each surgery was less than 90

Copyright (C) 2015, Iranian Society of Regional Anesthesia and Pain Medicine(ISRAPM). This is an open-access article distributed under the terms of the Creative Commons Attribution-NonCommercial 4.0 International License (http://creativecommons.org/licenses/by-nc/4.0/) which permits copy and redistribute the material just in noncommercial usages, provided the original work is properly cited. 
minutes. Consent was obtained from all patients. The variables of demographic features, onset time of the sensory and motor block, and the duration of sensory and motor block were answered in the questionnaire. The study was approved by the Ethical Committee of the Guilan University of Medical Sciences with ethical number 558 .

Patients with a history of allergy to the drugs, cardiovascular disorders, hypertension, coagulation abnormalities, excessive bleeding, $\mathrm{Hb}<10$, renal failure, skin infections around the axillae, opium addiction, pregnancy, or using beta blockers or calcium channel blockers were excluded from the study, as they are risk factors for high risk operations. It was shown that all blocks were perfect and all patients were suitable candidates for the procedure. Of the 60 patients, none were excluded.

Using randomization, 60 medical packets were prepared, mixed, and then given to the nurse in the operating room. Patients eligible to participate in this study and who had given written informed consent were referred to the operating room nurse who distributed one packet to each patient. The patients were introduced to the doctor and the nurse then discarded each packet. A total of 30 packets, named A, consisted of lidocaine plus magnesium sulfate and normal saline and the other 30 packets, named $B$, consisted of lidocaine plus normal saline. The nurse gave one of the closed envelopes to each patient and referred them to the doctor. After giving one packet to each patient, the nurse was not allowed to use it again.

Patients were thus randomly allocated into two groups. Group A received magnesium sulfate $(5 \mathrm{mg} / \mathrm{kg}$ lidocaine plus $3 \mathrm{~mL}$ of $20 \%$ magnesium sulfate and diluted in $40 \mathrm{~mL}$ of normal saline solution) and group $B$ (control) received normal saline instead of magnesium sulfate $(5 \mathrm{mg} / \mathrm{kg}$ lidocaine plus $3 \mathrm{~mL}$ of normal saline and diluted in 40 $\mathrm{mL}$ of normal saline solution). The dose of magnesium sulfate was calculated based on previous studies (5-7). The primary investigator prepared the pharmaceutical components. The anesthesiology resident, who did not know about the pharmaceutical composition, injected the drugs. The method of injection as well as the pinprick test and Bromage test were explained to all patients.

During surgery, ECG and oxygen saturation level were monitored with a Novin S 1800 machine (Pooyandegan Rahe Saadat). The patient's arm on which the operation was performed was abducted to 90 degrees supine and flexed at the elbow joint to 90 degrees. The armpit was shaved and betadine was applied to the site as a topical antiseptic. The patients did not receive any sedatives before the axillary block. The axillary artery was palpated at a site situated in the proximal of axillae and the overlaying skin was anesthetized with $1 \mathrm{~mL}$ of $2 \%$ lidocaine (Caspian Company, Iran). The axillary plexus block was performed under transarterial method and $20 \mathrm{~mL}$ of local anesthetic was injected posterior and $20 \mathrm{ml}$ in anterior to the artery. The continuous aspiration and injection was taken to avoid intravascular injection. The musculocuta- neous nerve was blocked by $5 \mathrm{~mL}$ of lidocaine $2 \%$ within the body of coracobrachialis muscle. The musculocutaneous nerve that provides sensory input for the lateral forearm also was blocked (in two groups). Sensory block level was monitored at one-minute intervals, as the onset of the nerve block is rapid. Sensory block level was performed by testing dermatomes of the sensory regions of the radial, median, and ulnar nerves. The intensity of the motor block was assessed using the modified Bromage scale at one-minute intervals as follows:

o) Unable to move fingers;

1) Able to move fingers only;

2) Able to bend wrist; and

3) Full flexion of the elbow.

When the sensory and motor blocks were fully established, the surgery was started. The sensory and motor blocks were assessed by the pinprick test and the modified Bromage test from the initiation of the blocks until the end of anesthesia. Such tests are safe and cause no damage. The end of sensory block was defined by have pain sensation during the pinprick test. At the end of motor block, the patient had pain sensation during the pinprick test in all sensate areas and the patient had complete movement of their body. All these tests were assessed in the recovery room. The operating time was 90 minutes or less and recovery time was approximately 30 minutes. After taking the patients to the recovery room, questionnaires of demographic features were completed. The duration of sensory and motor blocks were followed when the patients were in the recovery room. Sensory and motor responsiveness was tested at 10-minute intervals.

After inducing anesthesia in the patients, hemodynamic monitoring was performed. The patients who had blood pressure or pulse rate variability were excluded from the study as well as any patients who were not completely blocked 30 minutes after performing the infusion. After the sensory and motor block was established, patients reporting pain received an injection of $25 \mathrm{mg}$ of pethidine and were excluded from the study. Patients who received IV drugs to control anxiety were also excluded from the study. In the study, no one had complications. Analysis was performed by descriptive statistics, the Chi-square test, and the t-test. Data was analyzed by the SPSS (version 16). A P value $<0.05$ was considered statistically significant.

\section{Results}

A total of 60 patients were included in the study with 30 patients having received lidocaine plus magnesium and the other 30 patients having received lidocaine plus normal saline. Table 1 shows the demographic features of the patients from this study. By using the t-test, there were no significant differences in the demographic data of the patients and differences in the mean age and sex in the two groups were not significant. $(\mathrm{P}=0.805$ and $\mathrm{P}=$ 1). All block randomization was done successfully. All the 
patients were stable based on hemodynamic variables. No patients had complications such as hypotension to control and none were excluded from the study.

The mean sensory block duration in lidocaine plus magnesium group was $248.83 \pm 18.36$ and in lidocaine plus normal saline was $204.67 \pm 22.62$ (Table 2). The mean motor block duration in lidocaine plus magnesium group was $207.0 \pm 16.64$ and in lidocaine plus normal saline was $147.33 \pm 21.52$ (both $\mathrm{P}<0.0001$ ). The duration of the sensory and motor block was significantly longer in patients who received magnesium sulfate in lidocaine (Table 2).

The mean onset of sensory block in lidocaine plus magnesium group was $15.5 \pm 3.79$ and the onset block in lidocaine plus normal saline was $10.33 \pm 4.13$ and showed significant differences $(\mathrm{P}<0.0001)$. (Table 2 and Figure 1 ) The mean onset of motor block in the lidocaine plus magnesium group was $20.66 \pm 4.09$ and the onset block in the lidocaine plus normal saline was $19.73 \pm 26.18$ and did not show any significant differences $(\mathrm{P}<0.848)$ and (Table 2 , Figures 2 and 3 ).

Table 1. Demographic Features of the Patients ${ }^{\text {a }}$

\begin{tabular}{|c|c|c|c|c|}
\hline Features & Lidocaine + Magnesium & Lidocaine + Normal Saline & $\mathbf{t}$ & PValue \\
\hline Age, $y$ & $35.6 \pm 11.61$ & $34.93 \pm 10.24$ & 0.248 & 0.805 \\
\hline \multicolumn{5}{|l|}{ Gender } \\
\hline Male & $8(26.7)$ & $8(26.7)$ & - & 1 \\
\hline Female & $22(73.3)$ & $22(73.3)$ & - & 1 \\
\hline Weight, kg & $65.12 \pm 13$ & $66.16 \pm 11.65$ & 0.235 & 0.786 \\
\hline Surgical time, min & $78.65 \pm 8.42$ & $75.87 \pm 10.45$ & 0.225 & 0.682 \\
\hline Tourniquet time, min & $67.45 \pm 7.52$ & $66.38 \pm 7.86$ & 0.218 & 0.678 \\
\hline \multicolumn{5}{|l|}{ Site of surgery } \\
\hline Hand & 7 & 10 & - & 1 \\
\hline Forearm & 23 & 20 & & \\
\hline
\end{tabular}

${ }^{\mathrm{a}}$ Data are presented as mean \pm SD. or No. $(\%)$.

Table 2. Comparison of Mean Changes in Sensory and Motor Block in the Two Study Groups a

\begin{tabular}{lcccc}
\hline Variable & Lidocaine + Magnesium & Lidocaine + Normal Saline & t & P Value \\
\hline Onset of sensory block, min & $15.5 \pm 3.79$ & $10.33 \pm 4.13$ & 5.04 & 0.0001 \\
Onset of motor block, min & $20.66 \pm 4.09$ & $19.73 \pm 26.18$ & 0.193 & 0.848 \\
Sensory block duration, min & $248.83 \pm 18.36$ & $204.67 \pm 22.62$ & 8.3 & 0.0001 \\
Motor block duration, min & $207.0 \pm 16.64$ & $147.33 \pm 21.52$ & 12.01 & 0.0001 \\
\hline
\end{tabular}

a Data are presented as Mean \pm SD.

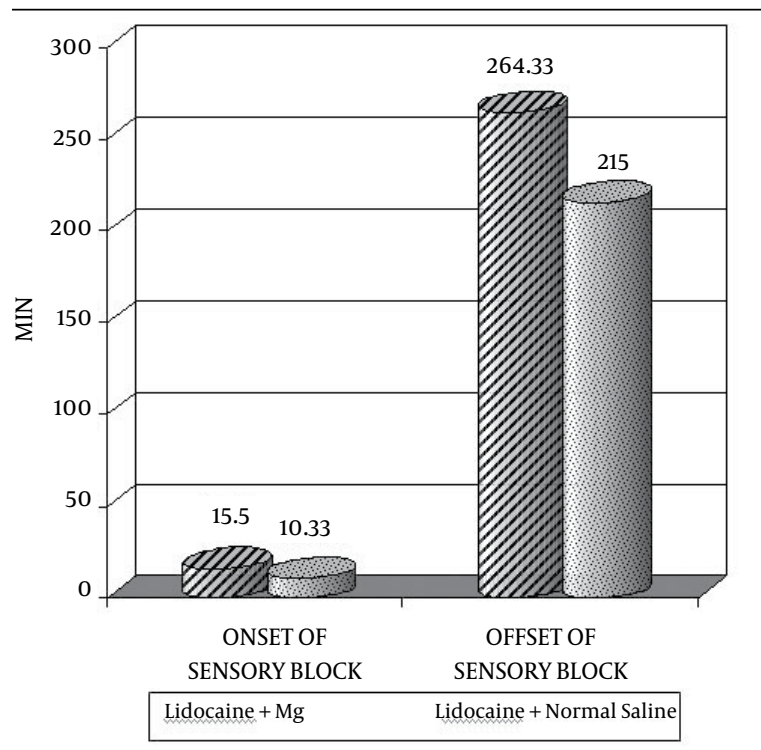

Figure 1. The Mean Onset and Offset of Sensory Block in the Two Groups

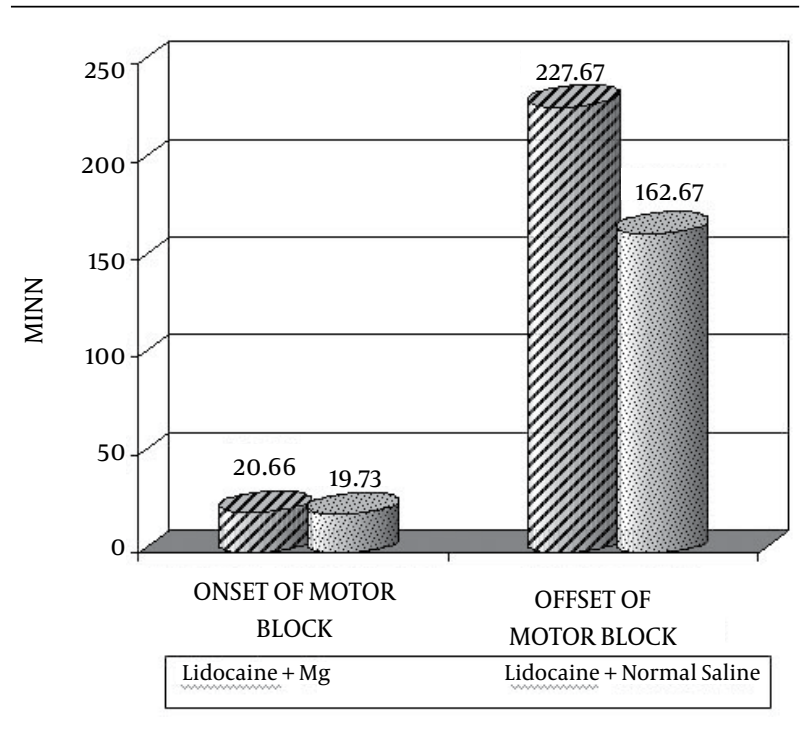

Figure 2. The Mean Onset and Offset of Motor Block in the Two Groups 


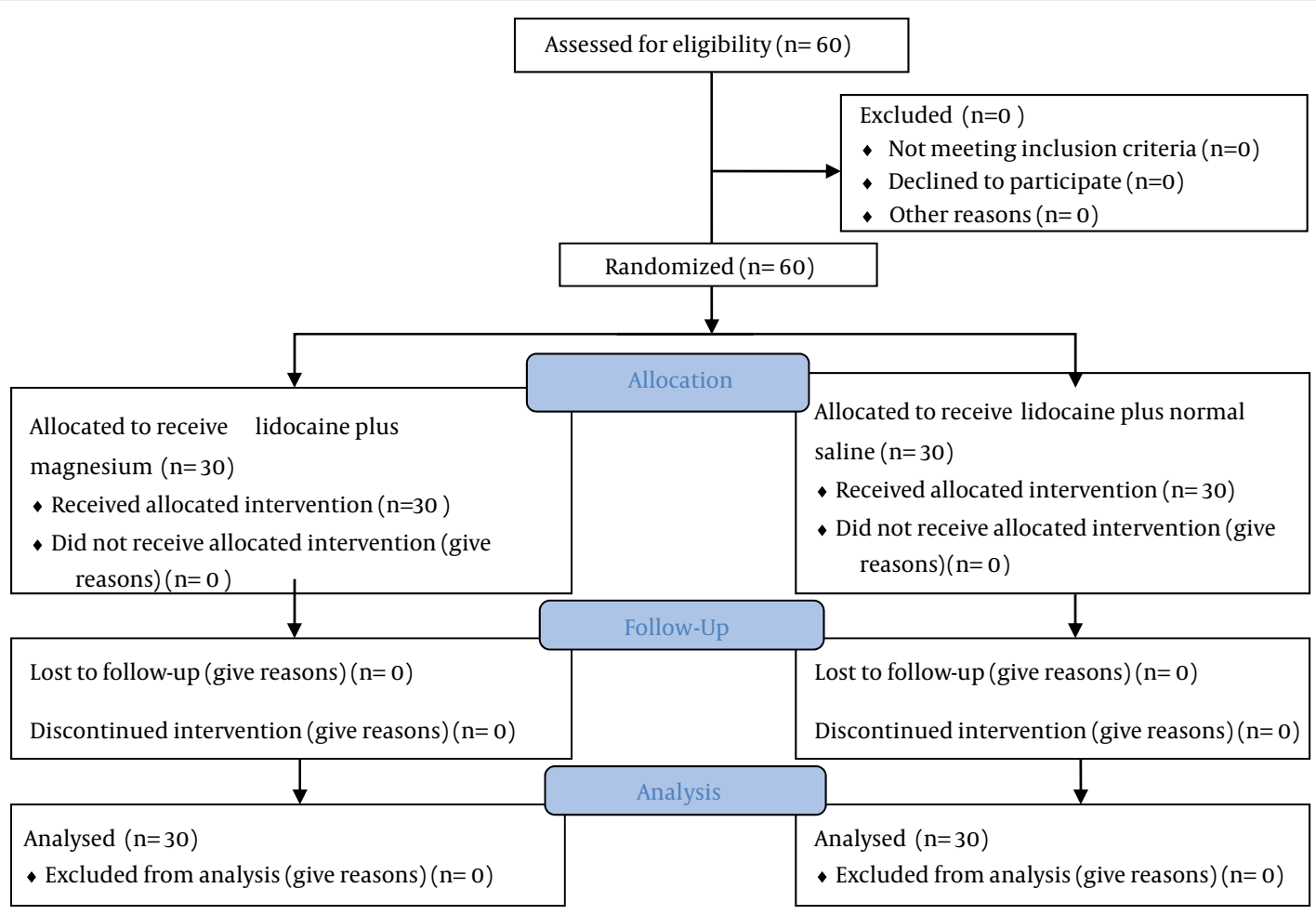

Figure 3. Flow Diagram

\section{Discussion}

Magnesium is a competitive NMDA-receptor antagonist and inhibits the voltage-dependent ion channels. Additionally, it seems that magnesium sulfate increases the length of block in intravenous regional anesthesia (8). Therefore, improving the quality of local anesthetics in regional and local blocks as well as the management of postoperative acute pain is important for patients who suffer from the pain after surgery such as orthopedic surgery. Various axillary block methods as well as anatomical differences in the nerves of the upper limb warrant further research. This study investigated the effects of adding magnesium sulfate with lidocaine in axillaryplexus blocks to prolong the duration of sensory and motor blockage and diminish postoperative acute pain for the patient.

In this study, the mean duration of the sensory and motor blocks in the lidocaine plus magnesium group was significantly longer than in the lidocaine plus normal saline group (control). We also assessed the onset of the sensory and motor blocks between the two groups. The onset time of sensory block, i.e., the time from when the infusion was completed and the first symptoms of anesthesia in the limb were established, was significantly longer $(\mathrm{P}<0.0001)$ for the lidocaine plus magnesium group than for the control group who received lidocaine plus normal saline group. The onset of anesthesia in the motor block also required a longer time in the magnesium plus lidocaine group when compared with the control group. However, this difference was not significant $(\mathrm{P}<$ 0.848). (Table 2) While an axillary block using lidocaine with magnesium sulfate has a delayed onset, the overall duration of the sensory and motor blocks was longer than the controlled condition of lidocaine with normal saline.

Ozalevli et al. compared intrathecal blocks of bupivacaine with fentanyl and magnesium in combination with sodium chloride in the patients undergoing lower limb surgery (9). They concluded that magnesium delayed the onset of sensory and motor blocks, but prolonged the duration of spinal anesthesia. In another study conducted by Elsharnouby et al. in Cairo in 2008, intra-articular injections of bupivacaine with magnesium resulted in longer periods of analgesia (duration) when compared with the control group that received a placebo (10). Two similar studies conducted by Arcioni et al. and by El-Kerdawy et al. reported that the addition of magnesium increased the duration time of an epidural analgesia $(11,12)$.

Narang et al. investigated the effect of magnesium in a Bier block and reported that the onset of sensory and motor analgesia was faster in the magnesium group than in the placebo group (13). There was also an increased incidence of transient pain on injection when magnesium sulfate was added to the block. These findings were in contrast to our study. Gunduz et al. reported that magnesium prolonged the duration of the axillary plexus block (5). Also in the study conducted by Abdelfatah et al. it was 
shown that magnesium sulfate plus lidocaine in an interscalene plexus block prolongs the analgesic duration in shoulder arthroscopic surgery, which was also consistent with our study (14).

One of the limitations of our study was a lack of regional anesthesia under guidance from sonography performed on the blocks. Therefore, it is recommended to perform another study to use above the technique. In addition, we recommend studying another sample size to receive higher doses of magnesium.

In the future, we recommend studying a larger sample size and follow up with patients for the longitude duration to confirm the results of the study and to assess the long-term complications of the drugs.

\section{Acknowledgements}

We gratefully acknowledge all volunteers, nurses, and doctors who were involved in this study.

\section{Authors' Contributions}

Study concept and design: Mohammad Haghighi; acquisition of data: Abbas Sedighinejad; analysis and interpretation of data: Mehdi Rahmati; drafting of the manuscript: Ahmadreza Mirbolook; critical revision of the manuscript for important intellectual content: Bahram Naderi Nabi; statistical analysis: Nasim Ashoori Saheli; administrative, technical, and material support: Mehran Soleymanha, study supervision: Mohammad Haghighi.

\section{References}

1. Kroin JS, McCarthy RJ, Von Roenn N, Schwab B, Tuman KJ, Ivankovich AD. Magnesium sulfate potentiates morphine antinociception at the spinal level. Anesth Analg. 2000;90(4):913-7.

2. Mesbah Kiaee M, Safari S, Movaseghi GR, Mohaghegh Dolatabadi MR, Ghorbanlo M, Etemadi M, et al. The effect of intravenous magnesium sulfate and lidocaine in hemodynamic responses to endotracheal intubation in elective coronary artery bypass grafting: a randomized controlled clinical trial. Anesth Pain Med.
2014;4(3).

3. Choi IG, Choi YS, Kim YH, Min JH, Chae YK, Lee YK, et al. The Effects of Postoperative Brachial Plexus Block Using MgSO(4) on the Postoperative Pain after Upper Extremity Surgery. Korean J Pain. 2011;24(3):158-63.

4. Buvanendran A, McCarthy RJ, Kroin JS, Leong W, Perry P, Tuman KJ. Intrathecal magnesium prolongs fentanyl analgesia: a prospective, randomized, controlled trial. Anesth Analg. 2002;95(3):661-6.

5. Gunduz A, Bilir A, Gulec S. Magnesium added to prilocaine prolongs the duration of axillary plexus block. Reg Anesth Pain Med. 2006;31(3):233-6.

6. Apan A, Buyukkocak U, Ozcan S, Sari E, Basar H. Postoperative magnesium sulphate infusion reduces analgesic requirements in spinal anaesthesia. Eur J Anaesthesiol. 2004;21(10):766-9.

7. Dabbagh A, Elyasi H, Razavi SS, Fathi M, Rajaei S. Intravenous magnesium sulfate for post-operative pain in patients undergoing lower limb orthopedic surgery. Acta Anaesthesiol Scand. 2009;53(8):1088-91.

8. Mirkheshti A, Aryani MR, Shojaei P, Dabbagh A. The Effect of Adding Magnesium Sulfate to Lidocaine Compared with Paracetamol in Prevention of Acute Pain in Hand Surgery Patients Under Intravenous Regional Anesthesia (IVRA). Int J Prev Med. 2012;3(9):616-21.

9. Ozalevli M, Cetin TO, Unlugenc H, Guler T, Isik G. The effect of adding intrathecal magnesium sulphate to bupivacaine-fentanyl spinal anaesthesia. Acta Anaesthesiol Scand. 2005;49(10):1514-9.

10. Elsharnouby NM, Eid HE, Abou Elezz NF, Moharram AN. Intraarticular injection of magnesium sulphate and/or bupivacaine for postoperative analgesia after arthroscopic knee surgery. Anesth Analg. 2008;106(5):1548-52.

11. Arcioni R, Palmisani S, Tigano S, Santorsola C, Sauli V, Romano $S$, et al. Combined intrathecal and epidural magnesium sulfate supplementation of spinal anesthesia to reduce post-operative analgesic requirements: a prospective, randomized, doubleblind, controlled trial in patients undergoing major orthopedic surgery. Acta Anaesthesiol Scand. 2007;51(4):482-9.

12. El-Kerdawy H. Analgesic requirements for patients undergoing lower extremity orthopedic surgery-the effect of combined spinal and epidural magnesium. Middle East J Anaesthesiol. 2008;19(5):1013-25.

13. Narang S, Dali JS, Agarwal M, Garg R. Evaluation of the efficacy of magnesium sulphate as an adjuvant to lignocaine for intravenous regional anaesthesia for upper limb surgery. Anaesth Intensive Care. 2008;36(6):840-4.

14. Abdelfatah AM, Elshaer AN. The effect of adding magnesium sulfate to lidocaine in an interscalene plexus block for shoulder arthroscopic acromioplasty. Ain-Shams J Anaesthesiol. 2014;7:59-64. 